\title{
Le culte de la mort dans l'« État nouveau » espagnol (1936-1941)
}

Francisco Sevillano Calero

$\mathbf{L}^{\prime} \ll$ État nouveau », au sortir de la guerre civile espagnole, a encouragé l'institutionnalisation d'un culte des morts tombés au combat ou victimes de l'ennemi républicain. Francisco Sevillano Calero explique comment une nouvelle liturgie d'État est organisée autour des cadavres, restes mortuaires et dépouilles de ces morts élevés au rang de martyrs par les dirigeants et les fonctionnaires du régime franquiste. Il montre comment la politique investit et mobilise le sacré, empruntant de nombreux éléments aux rituels et aux lieux de l'Église catholique, dans le but de figer une mémoire officielle de la guerre civile, représentée comme l'acte de naissance d'une nation régénérée dans son opposition existentielle à l'anti-Espagne.

Les études récentes sur le fascisme en Italie et le national-socialisme en Allemagne ont interprété le fascisme comme une forme moderne de religion politique ${ }^{1}$. Cette analyse n'a guère été appliquée par les chercheurs au cas de l'État nouveau espagnol. Les comparaisons entre le fascisme européen et la dictature franquiste ont principalement souligné l'influence fondamentale que l'Église catholique et la doctrine

(I) Selon le politologue italien Emilio Gentile, le fascisme possède, depuis ses origines, les caractéristiques d'une religion séculaire (voir « Fascism as Political Religion », fournal of Contemporary History, 25 (2-3), mai-juin 1990, p. 229-251). Cette thèse avait été exposée par George L. Mosse dans «Fascism and the French Revolution », fournal of Contemporary History, 24, 1989, p. 5-26. du national-catholicisme eurent dans le droit et la vie politique en Espagne ${ }^{2}$. Ce sont principalement les études des cérémonies, telles que le transport des restes mortels du fondateur de la Phalange 3 José Antonio Primo de Rivera, entre Alicante et le monastère de l'Escorial en novembre 1939, qui ont montré comment l'exaltation nationaliste adopta aussi les formes d'une religion politique séculière en Espagne ${ }^{4}$. Évoquons aussi les travaux montrant comment, sous la dictature, le souvenir

(2) La thèse de la «politisation du sacré » dans le franquisme a été exposée par Giuliana Di Febo dans Teresa d'Avila : un culto barocco nella Spagna franchista, 1937-1962, Naples, Liguri, 1988, 1987 ; trad. esp., id., La Santa de la Raza : un culto barroco en la España franquista, trad. de l'it. par Ángel SánchezGijón, Barcelone, Icaria, 1988, notamment p. 32-33 ; id., Ritos de guerra y de victoria en la España franquista, Bilbao, Desclée de Brouwer, 2002. Cette thèse a été défendue par Emilio Gentile dans Le religioni della politica : fra democrazie e totalitarismi, Rome, Laterza, 2001, p. 211-212 ; trad. fr., id., Les Religions de la politique : entre démocracies et totalitarismes, trad. de l'it. par Anna Colao, Paris, Éd. du Seuil, « La couleur des idées », 2005.

(3) Une note sur la Phalange figure en page 10 de ce numéro.

(4) Voir Eduardo González Calleja et Francisco Sevillano Calero, « Crociati moderni : dal lessico politico republicano alla propaganda franchista nella guerra spagnola $\gg$, Memoria e Ricerca : rivista di storia contemporanea, 11 (13), mai-août 2003, p. 89-113 ; Zira Box, « Pasión, muerte y glorificación de José Antonio Primo de Rivera », Historia del presente, 6, 2005, p. 191-218; id., «Rituales funerarios. Culto a los caídos y política en la España franquista : a propósito de los traslados de José Antonio Primo de Rivera (1939-1959) », dans Jesús Casquete et Rafael Cruz (dir.), Políticas de la muerte : usos y abusos del ritual fúnebre en la Europa del siglo XX, Madrid, La Catarata, 2009, p. 265-298. La thèse du franquisme comme religion politique a été défendue par Antonio Elorza Domínguez, « El franquismo, un proyecto de religión política », in Javier Tussell Gómez, Emilio Gentile et Giuliana Di Febo (dir.), Fascismo y franquismo cara a cara : una perspectiva bistórica, Madrid, Biblioteca nueva, 2004, p. 69-82. 
des victimes contribua à établir une politique officielle de la mémoire ${ }^{1}$.

En Espagne, le déclenchement de la guerre au cours de l'été 1936 a immédiatement confronté les populations à l'expérience traumatique de la mort de masse ${ }^{2}$. Le présent article analyse la transfiguration symbolique de la mort en un acte de sacrifice pour la rédemption de la patrie $^{3}$. Il explore ainsi la question d'une éventuelle sacralisation de la politique en Espagne par le culte rituel des « martyrs » et de ceux tombés au combat (caídos), se demandant si celui-ci fut l'expression d'une consécration de la nation. Le transfert des représentations chrétiennes de la mort, de la résurrection et du salut dans le domaine politique a permis

(I) Voir José L. Ledesma Vera et Javier Rodrigo, « Caídos por España, mártires de la libertad : víctimas y conmemoración de la guerra civil en la España posbélica (1939-2006) », Ayer : revista de bistoria contemporánea, 63, 2006, p. 233-255; Michael Richards, After the Civil War: Making Memory and Re-Making Spain since 1936, Cambridge, Cambridge University Press, 2013.

(2) Outre les travaux de Geoffrey Gorer, Philippe Ariès ou Michel Vovelle, publiés entre 1966 et 1975, sur les attitudes face à la mort en Occident, voir la critique de leur idée de la « mort inversée », c'est-à-dire la naissance du tabou de la mort au 20 siècle, par David Cannadine, « War and Death, Grief and Mourning in Modern Britain », in Joachim Whaley (dir.), Mirrors of Mortality : Studies in the Social History of Death, Londres, Europa Publications, 1981, p. 187-242. Les études du deuil collectif et l'utilisation politique de la mémoire des victimes se sont multipliées après le livre de Jay Winter, Sites of Memory, Sites of Mourning : The Great War in European Cultural History, Cambridge, Cambridge University Press, 1995. L'expérience collective de la Grande Guerre en France comme une expérience religieuse qui exacerba l'appréhension du divin par la présence massive de la mort a déjà été abordée par Annette Becker, La Guerre et la Foi : de la mort à la mémoire, 1914-1930, Paris, Armand Colin, 1994. Sur les cérémonies funéraires et la politique en France dans la perspective de la longue durée, voir Avner Ben-Amos, Funerals, Politics, and Memory in Modern France, 1789-1996, Oxford, Oxford University Press, 2000 ; trad. fr., id., Le vif saisit le mort : funérailles, politique et mémoire en France (1789-1996), trad. de l'angl. par Rachel Boyssou, Paris, Éd. de l'EHESS, 2013.

(3) Sur la conception du sacrifice comme ligne séparatrice entre le profane et le sacré, condition de l'existence divine, voir Henri Hubert et Marcel Mauss, « Essai sur la nature et la fonction du sacrifice », L'Année sociologique, 2, 1897-1898, p. 29-138, notamment p. 130 et p. 133 . Voir la compilation de ce travail dans Marcel Mauss, Euvres, Paris, Éd. de Minuit, 1968, t. I, p. $193 s q$. la sacralisation du souvenir à travers la rhétorique du culte funèbre pro patria mori, sa ritualisation et la consécration de lieux particuliers de mémoire. La mort pour la patrie fut vécue comme une communion spirituelle avec le corps mystique de la nation ${ }^{4}$. L'« État nouveau $\gg$ (Estado nuevo) ancra ainsi le culte officiel des morts dans une culture de guerre. Le souvenir des « martyrs » et des caídos se mêla au réseau de significations formé des valeurs, des idées, des symboles et des rites qui ont légitimé l'Alzamiento (littéralement « le soulèvement $»$, devenu nom propre pour désigner le coup d'État des 17-18 juillet 1936) et la « croisade $\gg$ contre l'anti-Espagne ${ }^{5}$.

Cette recherche s'étend chronologiquement de la guerre civile à l'après-guerre, de 1936 à 1940, en Espagne. Le parti unique, la Phalange espagnole traditionaliste et des juntes d'offensive national-syndicaliste (Phalange española tradicionalista y de las Juntas de ofensiva nacional sindicalista, FET et des JONS), et en particulier les secteurs des phalangistes les plus radicaux, capitalisèrent les célébrations

(4) Sur l'idée de mourir pour la patrie dans le monde chrétien médiéval, et son héritage dans le monde contemporain, voir Ernst H. Kantorowicz, « Pro Patria Mori in Medieval Political Thought », The American Historical Review, 56 (3), avril 1951, p. 472-492.

(5) Sur la notion de culture de guerre lors de la Première Guerre mondiale en France, voir Stéphane Audoin-Rouzeau et Annette Becker, «Vers une histoire culturelle de la Première Guerre mondiale », Vingtième Siècle. Revue d'bistoire, 41, janvier-mars 1994 , p. $5-7$; id., «Violence et consentement : la "culture de guerre" du premier conflit mondial », in JeanPierre Rioux et Jean-François Sirinelli (dir.), Pour une bistoire culturelle, Paris, Éd. du Seuil, 1997, p. 251-271. Cette notion a été précisée par ces auteurs dans d'autres publications, notamment 14-18, retrouver la Guerre, Paris, Gallimard, 2000. Cette approche, celle d'une histoire culturelle de la guerre, reprend l'interprétation de l'historien George L. Mosse sur la «brutalisation » de la politique dans l'Allemagne de l'après-guerre (George L. Mosse, Fallen Soldiers : Reshaping the Memory of the World Wars, New York, Oxford University Press, 1990). Pour un bilan sur l'importance de cette notion, voir Leonard V. Smith, «The "Culture de Guerre" and French Historiography of the Great War of 1914-1918 », History Compass, 5 (6), novembre 2007, p. 1967-1979 ; Pierre Purseigle, « A Very French Debate : The 1914-18 "War Culture" », Fournal of War and Culture Studies, 1 (1), 2008, p. 9-14. 
et les rituels de culte des morts. Cela suscita des réactions dans d'autres groupes politiques et au sein de l'autorité archiépiscopale de l'Église catholique espagnole ${ }^{1}$. Pour étudier comment le culte de la mort a été institutionnalisé dans cette période, diverses sources légales, testimoniales et documentaires ont été mobilisées, principalement la documentation originale du sous-secrétariat à la Presse et à la Propagande et celui de la Cause générale ${ }^{2}$ de Madrid.

\section{José Antonio Primo de Rivera, prophète de l'u Espagne nouvelle ॥}

Se superposant au temps liturgique de l'Église catholique, le culte des morts dans le calendrier festif de l'« État nouveau » débute avec la commémoration de l'assassinat de José Calvo Sotelo. Le décret du 10 juillet 1937 fit de l'anniversaire de sa mort, le 13 juillet, un jour de deuil national ${ }^{3}$.

L'exaltation des caídos pour l'Espagne a eu une très grande importance au sein de la Phalange et a constitué la principale expression

(I) Sur le conflit symbolique entre les secteurs politiques de l'État nouveau espagnol, les phalangistes, les monarchistes et les traditionalistes, voir Zira Box, España, año cero : la construcción simbólica del franquismo, Madrid, Alianza, 2010.

(2) Instruction, lancée en 1940 par le ministre de la Justice, des procès destinés à juger les délits commis dans les territoires restés sous gouvernement républicain après le soulèvement de 1936, et qui fut l'un des instruments de la répression franquiste.

(3) Boletín oficial del Estado (BOE), 12 juillet 1937. Le décret 253 du 12 avril 1937 avait établi le premier calendrier de festivités de l'« État nouveau » espagnol (BOE, 13 avril 1937). Le monarchiste José Calvo Sotelo (1893-1936) fut ministre des Finances sous la dictature de Miguel Primo de Rivera entre 1925 et 1930 . Déjà pendant la Seconde République, il fut élu député sous l'étiquette de Renovación española dans les élections en octobre 1933 et revint en Espagne de son exil au Portugal en mai 1934. Le 12 juillet 1936, José Castillo, un lieutenant de la garde d'assaut, membre du Parti socialiste ouvrier espagnol (Partido socialista obrero español, PSOE) et de l'Union militaire républicaine antifasciste (Unión militar republicana antifascista, UMRA), fut assassiné par un groupe de phalangistes à Madrid. Le lendemain, en représailles, des membres de la Garde d'assaut et des militants socialistes (appartenant au corps de protection du socialiste Indalecio Prieto Tuero) investirent la résidence de José Calvo Sotelo et l'emmenèrent avec eux pour le tuer à l'intérieur d'une fourgonnette de police. de son esthétique. Ainsi le 29 octobre 1937 futil célébré le Jour des caídos, lors de la Deuxième année triomphale (Segundo año triunfal, selon la terminologie officielle depuis le décret du 12 avril $1937^{4}$ ) de la guerre. Cette célébration coïncidait avec la fête de la fondation de la Phalange, parti créé en octobre 1933, et avec la fête des Jeunesses phalangistes. En vertu de l'arrêté du 5 février $1938^{5}$, le 9 février fut déclaré Jour férié de l'étudiant tombé (estudiante caído) dans tous les établissements d'enseignement, pour commémorer le souvenir du premier caído phalangiste, l'étudiant en médecine Matías Montero y Rodríguez de Trujillo. Cette célébration institutionnalisa un culte qui contribuait à ancrer les origines du soulèvement dans le désordre public et la violence politique de la période républicaine, et plaçait la jeunesse espagnole à l'avant-garde de l'Espagne nouvelle.

Le culte des caídos atteignit son expression maximale avec la construction du mythe de José Antonio Primo de Rivera, fondateur de la Phalange. Son exécution, à l'aube du 20 novembre 1936 à Alicante, l'a glorifié en un processus de « transfiguration par l'absence ${ }^{6} »$. Cette mythification commença par la transformation du mot «absent » («ausente ») en une devise officielle. En effet, comme l'a indiqué Dionisio Ridruejo : «l'adverbe a vite subi une nominalisation et José Antonio devint par définition l'absent ${ }^{7} \gg$. Le mythe de l'absence

(4) BOE, 13 avril 1937.

(5) BOE, 6 février 1938

(6) Cette expression fut utilisée par l'écrivain et homme politique Dionisio Ridruejo Jiménez, dans ses notes personnelles publiées à titre posthume sous le titre Casi unas memorias, Barcelone, Planeta, 1976, p. 174. Dionisio Ridruejo Jiménez (1912-1975), écrivain et homme politique espagnol, fut membre de la Phalange espagnole, ami et compagnon de José Antonio Primo de Rivera. En 1938, il fut nommé chef de la délégation nationale de la propagande du cabinet Franco.

(7) Ibid., p. 172. Selon Dionisio Ridruejo Jiménez, la devise s'est vraisemblablement formée par opposition au terme presente, employé de façon réitérée pour honorer les morts ou caídos. 
fut entretenu, comme tant d'autres, par les rumeurs confuses, jamais confirmées ni démenties, annonçant sa mort. Tout cela a contribué à la construction du mythe du fondateur de la Phalange et à la sacralisation de ses textes, de ses mots et de ses gestes ${ }^{1}$. Le général Franco annonça la mort de José Antonio Primo de Rivera lors d'une réunion de la Phalange tenue à Burgos, ville où le quartier général avait été déplacé au cours de l'année $1938^{2}$. Le décret du chef d'État du 16 novembre 1938 ordonna la commémoration de l'anniversaire de la mort de Primo de Rivera, considéré comme un des inspirateurs de l'«État nouveau », car ce dernier était issu de la guerre et d'une révolution nationale qu'il avait annoncées ${ }^{3}$. Primo de Rivera était devenu un héros national et le symbole du sacrifice de la jeunesse de cette période. Sa figure fut considérée comme prophétique en raison des pouvoirs visionnaires et du magnétisme que l'on attribuait à celui qui avait donné sa vie pour l'Espagne. Cette disposition légale a officiellement institutionnalisé le souvenir de Primo de Rivera dans le calendrier des célébrations et dans les lieux de mémoire. Le 20 novembre fut déclaré jour de deuil national. Auparavant, d'un commun accord avec les autorités ecclésiastiques, il avait été décidé qu'une inscription figurerait sur les murs de chaque paroisse avec les noms de ses

(I) « À bien des égards, écrit Dionisio Ridruejo Jiménez, cette mystification fut paralysante et transforma en simples scholiastes et glossateurs le peu d'hommes sensés - et je ne m'exclus pas - qu'a eue le phalangisme au cours de ces longues années. Elle a déclenché une bigoterie inhibitoire et a transformé le personnage en quelqu'un qui n'aurait peut-être pas été reconnu par ses inventeurs s'il était revenu - comme prévu - avec sa stature d'homme réel. » (Dionisio Ridruejo Jiménez, Casi unas memorias..., op. cit., p. 174)

(2) Pilar Primo de Rivera, Recuerdos de una vida, Madrid, DYRSA, 1983, p. 136. Dans ces mémoires, elle a également rappelé que la certitude de la mort de son frère encouragea Dionisio Ridruejo Jiménez à écrire une Corona de sonetos (Couronne de sonnets). Les différentes collaborations furent publiées sous le titre Corona de sonetos en honor de Fosé Antonio Primo de Rivera, s. l., Jerarquía, 1939.

(3) BOE, 17 novembre 1938. caídos « au cours de la croisade » ou « pendant la révolution marxiste ». De plus, ce décret prévoyait l'érection, à l'avenir, d'un monument dédié au souvenir de José Antonio Primo de Rivera. La portée de ses idées politiques serait expliquée et développée moyennant la création de chaires de doctrine politique dans les universités de Madrid et de Barcelone, appelées chaires José Antonio. Celles-ci ne seraient pas occupées par un titulaire fixe, mais par une succession de professeurs, nommés par le chef national de la Phalange et seraient subventionnées par des fonds du parti unique. Les services de presse et de propagande exalteraient cet héritage en organisant un concours national récompensant les meilleurs travaux artistiques, littéraires et doctrinaux consacrés à la figure et à l'œuvre de Primo de Rivera. Afin de perpétuer sa mémoire parmi les jeunes, les premières institutions à caractère national organisées pour la formation et la discipline politiques de la jeunesse et pour l'éducation artisanale des ouvriers porteraient son nom. L'Armée s'unissait également à l'hommage à Primo de Rivera en donnant son nom à une unité nouvelle de la marine de guerre, de l'armée de terre et de l'armée de l'air.

Un arrêté du ministère de l'Éducation nationale, du 16 novembre ${ }^{4}$, établit que le 22 du même mois, une leçon à la mémoire de José Antonio Primo de Rivera expliquant sa vie et son œuvre devait être dite dans toutes les écoles et les centres d'enseignement. La transcendance de la figure de Primo de Rivera était résumée dans le préambule de cet arrêté ministériel :

« La très haute personnalité de José Antonio Primo de Rivera, figure historique nationale, culmina dans sa mort héroïque : son dernier acte au service de l'Espagne.

Sa vie et son œuvre furent le chemin de cette prééminence, parvenant à l'accord spirituel et

(4) Ibid. 
humain le plus parfait : celui de la création qui prend racine dans l'âme de son peuple.

Ses paroles et son action favorisèrent dans notre patrie une façon de penser et d'être, point de départ de bon nombre de noyaux nationaux qui se soulevèrent lors des heures difficiles pour la cause sacrée de l'Espagne. »

Les funérailles de Primo de Rivera eurent lieu dans la cathédrale de Burgos le 20 novembre 1938. Auparavant, son nom avait été gravé sur les murs extérieurs du temple, près de la porte du Sarmental. Le Conseil politique de la Phalange avait décidé qu'il le serait dans toutes les églises d'Espagne. À la suite du nom de Primo de Rivera seraient inscrits ceux des fidèles, morts à la guerre dans le camp national ${ }^{1}$.

Ce 20 novembre, l'éditorial de l'édition sévillane du journal $A B C$ souligna que la mémoire de Primo de Rivera était évoquée « avec une émotion patriotique envers l'apôtre national et une émotion religieuse envers le martyr ${ }^{2} \gg$. Parmi les collaborateurs de ce numéro du journal, le directeur de l'Académie royale espagnole et conseiller national de la Phalange, José María Pemán, écrivit que la figure de Primo de Rivera constituait l'un des apports du Movimiento à la synthèse doctrinale nationale, ce qui l'érigeait en symbole majeur du régime, en prophète et en précurseur. Pemán jugeait ce legs conforme à la tradition catholique, loin de tout étatisme, du nationalisme et du paganisme, propres aux régimes totalitaires ${ }^{3}$.

(I) À l'imitation de ce qui s'était fait en France après 1918 (Dionisio Ridruejo Jiménez, Corona de sonetos..., op. cit., p. 175).

(2) $A B C$, Sevilla, 20 novembre 1938, p. 3

(3) José María Pemán, « José Antonio : pero todo José Antonio », $A B C$, Séville, 20 novembre 1938, p. 4-5. Le Movimiento nacional est la constitution en appareil politique du soulèvement militaire du 18 juillet 1936. Le général Francisco Franco prend la tête du mouvement comme Caudillo en octobre 1936. L'expression a servi à justifier l'acte d'unification politique dans le camp rebelle avec la formation du parti unique.
Le mythe de Primo de Rivera reflétait la présence du symbolique dans la politique au travers du rite et de la liturgie ; la mise en scène du transfert de sa dépouille depuis le cimetière d'Alicante en fut l'expression la plus intense et la plus organisée. Le 2 avril 1939, immédiatement après la guerre, Miguel Primo de Rivera se rendit dans cette ville pour récupérer dans une fosse du cimetière municipal le cadavre de son frère avec qui il avait été en prison. Les restes mortuaires furent déposés dans un coffre que l'on plaça dans une case du cimetière jusqu'à leur sépulture définitive ${ }^{4}$. Le 6 juillet de cette même année, le secrétaire général de la Phalange, Raimundo Fernández Cuesta, créa une commission pour dresser la liste des initiatives et établir les lignes concrètes de la cérémonie du transfert des restes mortuaires, autorisé par le Caudillo ${ }^{5}$. Le 9 novembre, la junte politique de la Phalange, présidée par Ramón Serrano Suñer, approuva ce transfert. Dionisio Ridruejo Jiménez suggéra que l'Escorial était l'endroit le plus approprié pour le repos de la dépouille, proposition qui se heurta à l'opposition de ceux qui considéraient le transfert au panthéon royal inopportun ${ }^{6}$. Par la loi du 15 novembre 1939, il fut décidé que les restes mortuaires seraient portés à dos de phalangistes depuis Alicante jusqu'à l'église du monastère de San Lorenzo de l'Escorial et que les honneurs de capitaine général lui seraient rendus lors de cérémonies se déroulant tout au long du transfert de sa dépouille et lors de son entrée dans sa nouvelle sépulture ${ }^{7}$. Ce fut la dernière des grandes célébrations de 1939,

(4) Le témoignage de Miguel Primo de Rivera a permis à Alfredo R. Antigüedad de raconter la période d'emprisonnement de Primo de Rivera à Alicante (Alfredo R. Antigüedad, Fosé Antonio en la cárcel de Alicante : un gran reportaje con Miguel primo de Rivera, Imp. de Ernesto Jiménez, s.l.n.d.).

(5) Boletín del Movimiento de Falange española tradicionalista y de las 7.O.N.S., 3 (60), 10 juillet 1939, p. 672.

(6) P. Primo de Rivera, Recuerdos de una vida..., op. cit., p. 148 .

(7) BOE, 17 novembre 1939. 
Année de la victoire (Año de la victoria) ${ }^{1}$. Elle réunit une foule nombreuse et se transforma en un rite de passage collectif, d'un lieu et d'un moment profanes en un lieu et un temps sacrés de l'Espagne nouvelle ${ }^{2}$.

\section{Exhumations, réinhumations et monuments aux morts}

La fin de la guerre civile, qui fut proclamée par le dernier communiqué du quartier militaire du généralissime, Francisco Franco, la nuit du $1^{\text {er }}$ avril 1939, samedi saint, a exacerbé le culte des morts. Ce cycle rituel de passage débuta par la récupération, le transfert et l'inhumation des restes des défunts honorés par le régime. Outrepassant l'autorité ecclésiastique sur les lieux sacrés, les organismes de l'« État nouveau » franquiste intervinrent dans le processus, le respect et l'hommage aux victimes au nom de la cause nationale (causa nacional). La loi du 16 mai 1939 permit aux municipalités de dispenser ou de réduire les taxes municipales sur les inhumations, les exhumations et les transferts des cadavres des victimes de la « barbarie rouge $\gg$ ou des morts au front ${ }^{3}$. Cela fut

(I) Les funérailles du fondateur de la Phalange espagnole sont narrées dans Samuel Ros et Antonio Bouthelier, $A$ bombros de Phalange : historia del traslado de los restos de fosé Antonio, Barcelone, Ediciones patria, s.d. [1940]. Sur la propagande par la diffusion de l'image de José Antonio Primo de Rivera dans le documentaire Presente!, qui filma des séquences du transfert de sa dépouille mortelle, voir Vicente Sánchez Biosca, « El Ausente, iPresente! : el carisma cinematográfico de José Antonio Primo de Rivera, entre líder y santo », Archivos de la Filmoteca, 46, 2004, p. 66-87.

(2) Sur cette notion, voir Arnold Van Gennep, Les Rites de passage, Paris, Éd. Nourry, 1909, éd. aug. 1969 : trad. esp., id., Los ritos de paso, trad. du fr. par Juan Aranzadi, Madrid, Taurus, 1986. Voir notamment le huitième chapitre sur les funérailles.

(3) BOE, 17 mai 1939. Les arrêtés du $1^{\text {er }}$ mai et du 22 juillet 1940, du ministère de l'Intérieur $(B O E, 9$ mai 1940 et 25 juillet 1940) précisent que les exhumations et inhumations des personnes ayant été assassinées dans la même province seraient autorisées par le préfet (gobernador civil), et que, si elles devaient se produire dans une autre province, elles le seraient par la direction générale de la Santé, les familles étant dispensées du paiement des droits sanitaires. Cependant, cette justifié dans le bref préambule de la loi, par le caractère exceptionnel du moment et la nécessité de rendre hommage aux «martyrs » et aux caídos, « victimes assassinées dans des circonstances tragiques ou mortes sur le front et ayant reçu une sépulture dans des endroits non appropriés ».

Le besoin de permettre aux familles, sur le plan administratif, la récupération, le transfert et l'enterrement des cadavres se superposa à la politique du souvenir des morts promue par le phalangiste Dionisio Ridruejo Jiménez depuis le sous-secrétariat à la Presse et à la Propagande. Pour garantir la solennité et le respect du souvenir lors des cérémonies publiques, ce soussecrétariat rédigea le 24 juin un arrêté réglementant l'exécution de l'hymne national dans certains spectacles et la transmission d'un communiqué quotidien des noms des caídos par la Radio nationale d'Espagne (Radio nacional de España). Le contraste fréquent entre ces solennités et le caractère frivole du spectacle conduisit bientôt à ce que les radiodiffusions du communiqué des caídos et l'hymne national ne soient plus obligatoires et que l'application de cette disposition soit laissée à l'appréciation des gouverneurs civils et des chefs provinciaux de la Propagande. Cependant, l'exécution des premières mesures de l'hymne national subsista à la fin de tous les spectacles, une fois le rideau baissé ${ }^{4}$.

Pour unifier le style des monuments commémoratifs de la guerre érigés en l'honneur des martyrs et pour sélectionner les projets de construction (qui pouvaient susciter un enthousiasme incontrôlable, mais aussi des déceptions quand ils n'étaient pas viables), l'arrêté du

disposition n'a pas affecté les éventuelles décisions judiciaires interdisant ou conditionnant les exhumations de victimes de faits délictueux.

(4) Archivo general de la administración (AGA), Sección cultura (SC), Ministerio de Información y Turismo (MIT), c. 1378 . 
ministère de l'Intérieur du 7 août établit que ces projets, ainsi que l'ouverture de souscriptions pour leur mise en œuvre, devaient préalablement être soumis à l'approbation ministérielle. De même, la publication de nouvelles ou de rapports, ou de toute autre forme de propagande sur ce type de projets fut interdite jusqu'à ce que soit obtenue l'autorisation ministérielle par l'intermédiaire du chef du service national de la Propagande ${ }^{1}$.

Les initiatives pour la récupération des corps des victimes, le décorum en leur honneur et la perpétuation de leur souvenir par des monuments commémoratifs servirent à établir dans tout le pays une mémoire officielle du passé au travers du culte des morts. Le sacrifice de la mort, vécu rituellement de façon périodique comme un phénomène religieux collectif, permit au profane de communiquer avec le sacré : la nation en tant que communauté imaginée. Ce culte politique profondément nationaliste, et profane dans quelques-unes de ses manifestations, occupa l'espace public, et fit également irruption dans les lieux sacrés de l'Église catholique. Il utilisa les symboles, les rites et les célébrations liturgiques du souvenir des victimes qui avaient fait preuve de leur foi en la patrie en mourant des mains des ennemis de l'Espagne.

(I) BOE, 22 août 1939. Pour répondre aux demandes adressées au ministère, accompagnées du rapport préalable des autorités intervenant dans la procédure, il fut établi, le 5 octobre de cette même année, qu'il reviendrait à la direction générale de la Propagande d'autoriser les monuments, pierres tombales, inscriptions, etc., et à la direction générale de la Bienfaisance ce qui concernait les souscriptions (AGA, SC, MIT, c. 1364). Quelques jours plus tard, le 16, il fut établi que les préfets transféreraient au sous-secrétariat à la Presse et à la Propagande du ministère de l'Intérieur toutes les initiatives de construction de monument, avec leur rapport. Le sous-secrétariat remettrait à la direction générale de la Propagande cette documentation, et à la direction générale de la Bienfaisance les demandes de souscriptions pour la construction de ces monuments, appels à projets, etc. (AGA, SC, MIT, c. 1378). L'arrêté du 30 octobre 1940 réglementa le traitement des dossiers sur les initiatives de commémorations (BOE, 12 novembre 1940).

\section{Les lieux centraux du culte des morts}

Le souvenir des «martyrs » et des caídos dans les tombes et les cimetières établit un point fixe qui situe la mémoire collective. Le cérémonial, en tant qu'expérience rituelle collective de l'espace et du temps sacrés, est l'acte médiateur entre la mémoire collective, la mémoire officielle et la mémoire nationale que l'État veut établir en toute société. Dans ce but, le site de Paracuellos de Jarama, près de Madrid, fut placé au centre de la politique mémorielle de l'« État nouveau » espagnol. À l'occasion du troisième anniversaire du début des exécutions de prisonniers par les républicains les 6 et 7 novembre 1936 dans cette commune, le chef provincial de la Phalange de Madrid, Jaime de Foxá Torraba, communiqua au sous-secrétariat à la Presse et à la Propagande, le 28 octobre, les cérémonies programmées. Dans la fosse où reposaient les corps des «martyrs », on veillerait la nuit du 6 au 7 novembre, sous la garde des milices madrilènes de la Phalange. Le 6, une messe serait célébrée à minuit avec accord de l'évêché et, pendant toute la nuit, d'autres messes seraient dites jusqu'à neuf heures du matin, moment où une messe avec communion générale serait officiée. La prière des caídos (oración de los caídos) serait lue et le cri rituel « présents! $!($ presentes! $!)$ pour les caídos serait prononcé. Le 7 , des messes seraient célébrées dans toutes les églises de Madrid pour le repos éternel de leurs âmes. Le chef provincial suggéra que le ministre Ramón Serrano Suñer, en tant qu'ex-prisonnier, intervienne à la radio la nuit du 6 afin de rappeler cette date et demande au peuple madrilène de se rendre à la fosse de Paracuellos de Jarama « pour déposer leurs prières et leurs fleurs sur ce lieu tragique ${ }^{2} »$.

Quelque temps auparavant, le 17 octobre, le sous-secrétaire du ministère de l'Intérieur avait communiqué à la direction générale

(2) AGA, SC, MIT, c. 1346. 
de la Propagande une requête de Luis Pérez Izquierdo, capitaine de corvette de la marine de guerre et délégué à Burgos de l'Association des familles des martyrs de Paracuellos de Jarama et de Torrejón de Ardoz (Asociación de Familiares de Mártires de Paracuellos de Jarama et de Torrejón de Ardoz). Celui-ci avait demandé au ministre de proposer au Conseil des ministres une souscription pour ériger un monument perpétuant la mémoire de ceux ayant « donné leurs vies pour l'Espagne » à Paracuellos de Jarama et Torrejón de Ardoz. Le chef de l'État Francisco Franco contribuait personnellement à cette souscription avec un apport de cent mille pesetas ${ }^{1}$. La contribution du gouvernement fut fixée à vingt-cinq mille pesetas par ministère.

Peu après, le 8 novembre 1936, l'amiral Francisco Bastarreche, président du comité de direction de l'association des familles, écrivit au directeur général de la Propagande, qu'étant donné « la signification si forte de ce champ de chevaliers immolés pour Dieu et pour leur patrie $^{2} »$, il était urgent et indispensable de construire, dans le délai le plus bref possible, un autel pour le cimetière de Paracuellos de Jarama, afin d'y remplacer la table en bois sur laquelle était dite la messe. Un projet et un devis furent rapidement établis par la direction générale de la Propagande et soumis au président de l'Association des familles des martyrs de Paracuellos de Jarama et de Torrejón de Ardoz.

Le décret du 3 février 1940 permit aux dépouilles des Espagnols assassinés à Torrejón de Ardoz de recevoir les honneurs de capitaine général ${ }^{3}$. Le 12 février, l'amiral Francisco Basterreche envoya au directeur général de la Propagande une copie du cortège et de l'itinéraire à suivre jusqu'au lieu où serait célébrée,

\footnotetext{
(I) AGA, SC, MIT, c. 5372.

(2) AGA, SC, MIT, c. 5372.

(3) $\mathrm{BOE}, 6$ février 1940.
}

le dimanche 18 février, la cérémonie qui rendrait, selon les ordres du chef de l'État, les honneurs aux dépouilles. Celles-ci, qui venaient d'être exhumées, représenteraient symboliquement tous les caídos de Madrid et de sa province $^{4}$.

Du 7 au 20 décembre 1939, l'Association des familles avait procédé à l'exhumation des dépouilles mortelles dans la commune de Torrejón de Ardoz ; 414 cadavres avaient été récupérés, dont 64 purent être identifiés ${ }^{5}$. Lors des funérailles du 18 février 1940, les cadavres provenaient des exhumations réalisées à Soto de Aldovea et à Torrejón de Ardoz, d'où ils furent conduits au cimetière de la Almudena. Les six cadavres étaient ceux d'Ildefonso Álvarez de Toledo, marquis de Villanueva de Valduesa ; Luis Cervera Jácome, capitaine de navire ; Enrique Pérez Izquierdo, commandant d'artillerie ; Eduardo Vilades Abadía, pilote aviateur et deux personnes non identifiées. À dix heures et demie du matin, les camions transportant les cercueils arrivèrent. Les autorités militaires les prirent en charge, les disposant sur des caissons d'artillerie, les couvrant du drapeau national pendant qu'une batterie tirait les salves d'artillerie. Différentes personnalités et délégations portaient les bandes de chaque cercueil. À droite du cortège funèbre se trouvaient le chef de la première région militaire et, à la suite, une délégation du clergé madrilène, la croix levée ; derrière chaque caisson marchaient deux infirmières de guerre portant des bouquets d'œillets rouges et jaunes ainsi que des rubans portant l'inscription « hommage à nos martyrs ». Ensuite se trouvait la direction de l'Association des familles, présidée par l'amiral Basterreche. De nombreux marins suivaient, la compagnie d'honneur formée des

(4) AGA, SC, MIT, c. 5372.

(5) Archivo histórico nacional (AHN), Fondos contemporáneos (FC), Causa general (CG), 1. 1536, exp. 2, « Pieza especial de Madrid sobre exhumaciones de mártires de la cruzada ». 
forces militaires aux côtés d'une centurie de la Phalange et une autre de l'Organisation de la jeunesse, tandis que les ministres de la Marine de guerre, de l'Armée, de la Justice et des Finances et diverses délégations fermaient la marche.

Lorsque le cortège arriva devant l'autel, les cercueils furent placés sur des tombeaux et une messe pour les corps sans sépulture (corpore insepulto) fut célébrée. Une fois terminée, un répons fut chanté et un avion lâcha des fleurs sur les cercueils. Les forces armées défilèrent devant les cadavres en colonne d'honneur. Quand le défilé s'acheva, deux escadrons d'aviation survolèrent les lieux en formant une grande croix et lachèrent de nouveau des fleurs. Devant la sépulture définitive des six dépouilles, l'amiral Basterreche lut la prière des caídos, en lançant le cri rituel « martyrs de Madrid et de sa province !». À la suite de quoi, tous répondirent « présents! $\gg$ Des salves de fusils furent tirées, et avant de lancer de la terre sur les cercueils, José María Pemán fit l'éloge funèbre des caídos pour Dieu et pour l'Espagne, affirmant que ceux-ci ainsi que les « martyrs » formaient les deux piliers sur lesquels l'Espagne nouvelle devait se fonder.

Le sacrifice des victimes au nom de la patrie était la matrice des croyances de la communauté nationale, de sa conscience nationale. Léditorial de La Vanguardia española du mardi 20 février souligna que la cérémonie avait constitué un serment tacite pour la permanence de l'héritage de ces martyrs morts pour la patrie, « rendant éternelle, avec le souvenir du crime sans nom, la volonté tendue et irrévocable de défendre l'Espagne contre tous les ennemis plus ou moins larvés de la pensée et de l'amour de l'holocauste au cours duquel ces compatriotes héroïques donnèrent leur sang généreux ${ }^{1} \gg$. Dans toute l'Espagne, chaque

(I) La Vanguardia española, 20 février 1940. cérémonie funèbre célébrée en hommage à ceux qui étaient tombés, constituait, comme le souligna également l'éditorial de l'édition madrilène d' $A B C$ le jour suivant, une « promesse de vie dans une réaffirmation profonde du présent $\gg^{2}$. L'évocation pieuse du sacrifice des caídos devait être interprétée comme une leçon de courage pour l'Espagne future. Chaque fosse devait être un exemple et non un simple symbole funéraire. « Nous avons l'engagement de former une patrie telle qu'ils la défendirent avec leur foi face aux piquets d'exécution. »

Le souvenir du culte des victimes enterrées dans la fosse du site du ruisseau de San José, à Paracuellos de Jarama, montre que le geste rituel engendre la mémoire en faisant du passé une réalité vécue de façon ininterrompue dans le présent. La présence de la mémoire dans la société réactualise un événement, en fait un symbole pour une communauté. Cette configuration symbolique d'une identité nationale est à l'origine d'un processus d'intégration et de désintégration politiques, par l'instauration d'une division entre amis et ennemis.

Le décret du $1^{\text {er }}$ avril 1940 stipula le choix, comme lieu de repos pour « ceux qui tombèrent lors de notre croisade », des versants de la sierra de Guadarrama, connus sous le nom de Cuelga-muros et situés à neuf kilomètres et demi au nord du monastère de San Lorenzo de l'Escorial, et la construction urgente d'une basilique, d'un monastère et d'une caserne pour les Jeunesses ${ }^{3}$. Selon le préambule justificatif du décret, ce choix correspondait à l'importance de la croisade, à l'héroïsme des sacrifices qu'avait supposés la victoire et à la

(2) « El mandato de los que cayeron », $A B C$, Madrid, 21 février 1940.

(3) BOE, 2 avril 1940. Cette construction d'un lieu religieux n'a fait l'objet d'aucun consentement préalable, par écrit, de l'autorité ecclésiastique, contrairement à ce que prévoyait le Code de droit canonique alors en vigueur (Codex Iuris Canonici, IX, c. $1162 s q$. , dans AAS, IX, pars II : $226 s q$.). 
transcendance de cette épopée pour le futur de l'Espagne. Tout cela ne pouvait être perpétué par de simples monuments commémoratifs érigés dans les villes.

« Il est nécessaire que les pierres qui seront érigées aient la grandeur des monuments anciens, qui défient le temps et l'oubli et constituent un lieu de méditation et de repos où les générations futures rendront hommage et admiration à ceux qui leur léguèrent une Espagne meilleure.

À ces fins répond le choix d'un lieu isolé où se dresse le temple grandiose de nos morts, où pendant des siècles on puisse prier pour ceux qui sont tombés sur le chemin de Dieu et de la patrie. Lieu éternel de pèlerinage où le caractère grandiose de la nature soit un cadre digne pour le repos des héros et des martyrs de la croisade. »

Francisco Franco, en tant que président du gouvernement, encouragea ce projet de panthéon par le biais du Conseil des travaux du monument aux caídos (Consejo de obras del monumento nacional del valle de los Caídos), Conseil crée par décret en juillet $1941^{1}$. Ce fut un geste non vers la réconciliation, mais pour la perpétuation du souvenir de la « croisade $\gg$ et des caídos. Ainsi fut prévu le transfert au Panthéon des caídos des dépouilles des victimes non identifiées, qui auraient souffert sous la «domination rouge ». L'arrêté du 4 avril de cette année soulignait la diversité des lieux où la « fureur marxiste » avait mené ses victimes pour leur donner la mort ${ }^{2}$. Dans beaucoup de

(I) BOE, 6 août 1941 .

(2) BOE, 5 avril 1940. Un nouvel arrêté du 11 juillet 1946 (BOE, 15 juillet 1946) reporta indéfiniment les enterrements temporaires des dépouilles des caídos, qu'ils aient succombé dans le camp de « l'Armée nationale » ou qu'ils aient été assassinés par les « hordes marxistes », et leva l'obligation de transférer les dépouilles mortelles à la fosse commune si, passés dix ans, un enterrement définitif n'avait pas été acquis. La construction de la crypte qui, dans la vallée des Caídos, offrirait une sépulture digne aux « héros et martyrs de la croisade » éviterait que, par manque de moyens ou du fait de la négligence de leurs proches, des dépouilles se perdent. communes, des lieux étaient ainsi apparus, où se trouvaient des dépouilles humaines dont l'identification était impossible et qui n'avaient pas été réclamées par les familles. L'hommage exigeait que, jusqu'à ce que les dépouilles puissent être recueillies dans le Panthéon des caídos, des mesures soient prises pour éviter de possibles profanations et pour assurer le respect qui leur était dû. Les municipalités limiteraient et fermeraient de façon provisoire les lieux où l'on était sûr que des dépouilles de personnes assassinées par les « rouges » n'avaient pas été réclamées par leurs familles. Après avoir délimité le lieu, la municipalité demandait à l'autorité ecclésiastique qu'elle lui confère le caractère de terre sacrée, comme s'il s'agissait d'un nouveau cimetière municipal. Si le nombre de caídos était très réduit, la mairie transférait les corps dans un espace spécifique au sein du cimetière le plus proche ; le texte précisait que celui-ci devait être à la mesure de la situation ${ }^{3}$.

Le culte des martyrs et des caídos dans l'« État nouveau » franquiste montre que l'exacerbation du sentiment nationaliste (encouragée émotionnellement par les représentations de la guerre, dans le cadre d'une vision cohérente du passé, du présent et du futur) permit un transfert de sacralité à la politique par l'élaboration d'une théologie et d'une religion politique. Le culte des morts fut un élément important qui contribua, en tant qu'expérience collective d'union avec le sacré, à la transfiguration de la nation espagnole en une entité spirituelle. La

(3) Du fait des exhumations de victimes réalisées au cours de l'instruction de la Cause générale (Causa general) depuis mai 1940 par le procureur du Tribunal suprême pour recueillir des preuves des délits commis durant la «domination rouge », environ 826 dépouilles de la province de Madrid (surtout dans les communes de Torrejón de Ardoz, Boadilla del Monte et de Barajas), dont 123 identifiées, furent transférées vers la fin de l'année 1948 au cimetière de Paracuellos de Jarama en vue de leur inhumation dans la vallée des Caídos. (AHN, FC, CG, 1. 1536, « Pieza especial de Madrid sobre exhumaciones de mártires de la cruzada ») 
nation comme union sacrée résulte des souvenirs, des sacrifices, des gloires, des deuils et des peines communes du passé et de la volonté de faire valoir dans le présent, en tant que communauté, l'héritage reçu.

Cependant, le rituel politique du culte des victimes n'a pas servi seulement aux stratégies d'intégration nationale car, de manière ambivalente, il a aussi permis des stratégies symboliques différenciées pour assurer ou atteindre le pouvoir dans l'« État nouveau ». L'appropriation phalangiste du culte des caídos, non seulement perpétua le cérémonial antérieur de la Phalange espagnole en hommage à ses militants morts, mais elle situa aussi l'origine de l'«Espagne nouvelle » et la signification du sacrifice collectif de la guerre dans un moment primordial : la fondation du parti phalangiste lors de la cérémonie célébrée au théâtre de la Comedia, à Madrid, par José Antonio Primo de Rivera, le 29 octobre 1933. Cette figure, sanctifiée par son propre sacrifice, occupa le centre du culte des martyrs pour la patrie à partir de 1938. De cette façon, le catholicisme fut certes une religion pratiquée, mais aussi une culture reçue et servant de répertoire dogmatique, liturgique et rituel à la politique pour la légitimation d'un nouveau régime qui s'est confondu avec la nation espagnole.

Francisco Sevillano Calero, Université d'Alicante, 03690, San Vicente del Raspeig, Espagne.

Francisco Sevillano Calero enseigne l'histoire contemporaine à l'Université d'Alicante. Ses recherches portent sur la guerre civile espagnole et la dictature franquiste, et notamment sur la propagande politique. II est l'auteur, entre autres ouvrages, de Propaganda y medios de comunicación en el franquismo (Publicaciones de la Universidad de Alicante, 1998), Ecos de papel: la opinión de los españoles en la época de Franco (Biblioteca nueva, 2000), Exterminio : el terror con Franco (Oberon, 2004), Rojos : la representación del enemigo en la guerra civil (Alianza, 2007) et Franco : Caudillo por la gracia de Dios (Alianza, 2010). (fsevillano@ua.es) 